\title{
QUANTUM FIELD THEORY AND THE DYNAMICAL ROLE OF SPIN
}

\author{
FRANÇOIS LURÇAT* \\ Laboratoire de Physique Théorique et Hautes Energies, Orsay, France
}

(Received 20 June 1964)

\begin{abstract}
The point of view currently taken in elementary particle physics, that the spin is an unessential complication and plays no dynamical role, is criticized here. This assumption has no experimental ground; it is merely a consequence of the wave equations used, especially in field theory. To get rid of it, we must define wave functions and fields as functions, not on Minkovski space, but on the Poincare manifold (i.e., the manifold of the Poincaré group). The result is a "Poincare field theory," for which the general features of field theory (relativistic invariance, locality, definition of free fields) are formulated. The spectral condition is only sketched. A physically important result is a generalized Kallèn-Lehmann representation of the two-point function, where the spectral function depends on the mass and also on the spin. As a consequence, the peaks in this spectral function represent particles which may have different spin values, and (when they are unstable) a spin spectrum as well as a mass spectrum. The notion of Regge trajectory appears as a particular case.
\end{abstract}

\section{Introduction}

IN particle physics, it is a commonplace idea among theoreticians that the spin is an "unessential complication." More precisely, the spin turns out to be a purely kinematical feature of particles [1]; Lorentz invariance appears to be less important than translation invariance, polarizations less important than momenta, etc.

This idea may have its origin in the fact that we live in an "unpolarized" world; i.e., the atomic constituents of macroscopic bodies are, in general, practically not polarized. This applies especially to bubble chambers and other recording or counting devices; therefore, unless special techniques are used, the polarization effects are averaged out. But this has nothing to do with the properties of particles themselves, only with the thermodynamical fact that we do not fill bubble chambers with a ferromagnetic liquid. There does not exist any known property of particles showing that spin is less important than mass. Let us consider a chart of particles; they have masses and spins; the chart does not suggest that spin should be given a lower position then mass, but suggests studying the relations between masses and spins (for instance, by using the notion of Regge trajectories). Let us consider then a decay or a reaction process; both spins and masses are, in general, different before and after the process; nothing suggests that the spin change should be a less important fact than the mass change.

We would like to show in what follows that the spin turns out to be an unessential complication only because we force it to play this modest role. This happens when we postulate that particles can be described with a usual wave function, or a usual field, i.e., with a wave function or a field defined as functions on Minkovski space.

Let us analyze briefly the physical implications of this postulate. First, we consider non-relativistic classical mechanics of a point particle. The position of this particle is defined by a point in three-dimen-

*Postal address: Laboratoire de Physique Théorique et Hautes Energies, Bâtiment 211, Faculté des Sciences, Orsay (Seine-et-Oise), France. 
sional euclidean space. The physical meaning of a point particle is the following: we consider a small solid, and we go to the limit where all the dimensions of this solid tend to zero. But this limiting process is singular, because a solid has not three, but six degrees of freedom; the concept of a point particle implies not only zero dimension, but also neglect of the three rotational degrees of freedom. This neglect is justified dynamically, when the rotational (kinetic + potential) energy is much smaller than the translational energy. But this does not always happen. If it is not the case, we must take as configuration space for our solid, not the euclidean three-dimensional space, but the six-dimensional manifold, with points representing possible positions of the solid, i.e., of a coordinate system attached to it. It is easy to see that this manifold is isomorphic to the group of all displacements (translations and rotations) of the euclidean space (the so-called euclidean group of the three-dimensional space). Indeed [2], let us choose a coordinate system $R_{0}$ in the space; for any coordinate system $R$, with the same orientation as $R_{0}$, there is a unique element $g$ of the (connected) euclidean group, which sends $R_{0}$ onto $R$ :

$$
R=g R_{0} .
$$

This allows us to define a one-to-one correspondence between the $g$ 's and the $R$ 's; thus, each element $R_{0}$ of the "euclidean manifold" (i.e., the manifold of the euclidean group) defines a one-to-one correspondence of this manifold with the euclidean group.

We conclude that, unless we are allowed by the dynamical situation to neglect the rotational degrees of freedom of our small solid, its configuration space is not the three-dimensional space, but a manifold isomorphic to the euclidean group of the three-dimensional space.

Let us consider the Minkovski space. A point in this space represents an event; that is, the position of a small body at a given time, or rather during a small time interval. Let us suppose we cannot neglect the rotational degrees of freedom; this implies that we give, not only the position of the centre of the body, and of the "centre" of the time interval, but also the spatial directions of the three space axes attached to the body. To be covariant, then, we must give the space-time directions of the four space-time axes, which define, not only the space direction of the body, but also its "space-time direction," i.e., the velocity of its centre of mass. The same method as used above shows that the manifold of all the space-time coordinate systems, of given space and time orientations, is isomorphic to the connected inhomogeneous Lorentz group, which we shall call henceforth the Poincare group.

Thus, unless we are allowed to make a special "isotropy assumption," the natural frame of a relativistic theory is not Minkovski space, but a manifold isomorphic to the Poincare group [3] (see section II for a more precise statement). This manifold will be called the Poincare manifold.

Let us take as another example the (classical) electromagnetic field. To study this field, we use an apparatus (for instance a magnetometer) which measures the component of either the electric or the magnetic field, in a given space direction. The result of the measurement depends, not only on the position of the apparatus, but also on its space direction and its velocity. Hence, the complete description of the electromagnetic field is most naturally given by a (scalar-valued) function of the space-time coordinate system attached to the apparatus, or equivalently by a function on the Poincare manifold.

Passing now to wave equations, we may take as our starting point this remark of Wigner's, that when we write wave equations we force the wave function to be a function of $x$ [4]. Let us try to amplify this remark: to describe spin-zero particles, we use the Klein-Gordon equation, which writes in a natural way as an equation on the Minkovski space; but we have more trouble for non-zero spin. The procedure generally used is a very ingenious one: one takes a vector-valued wave function, with values in the vector space of a representation of the (homogeneous) Lorentz group [5]. This device, however, has two shortcomings. The first one is that there is an ambiguity in the choice of the representation of the Lorentz group: if we want to describe particles of spin $s$, we may choose any representation $\left(j, j^{\prime}\right)$ with $j+j^{\prime}=s$. (As physicists usually do, we label the representations of the Lorentz group by two integer or half-integer indices; see, for instance, reference 6). This ambiguity entails in general the appearance of redundant components of the wave function, which have to be eliminated by supplementary conditions. The situation is rather complicated, and there is no proof that this complication corresponds to some real property of the particles [7]. The second shortcoming is more essential: it appears when we pass from our wave equation to a quantum field theory modelled after it. Of course, unless we make the theory of a free field, we abandon the wave equation itself; this allows the particles associated with our field to go out of their mass shell. But we keep an essential feature of the wave equation: as the wave function, the field (or, more exactly, the matrix elements 
of the field operator) will be vector-valued, with values in the vector-space of some definite representation of the Lorentz group. This means that the way we force our field to describe particles with spin $s$ is a very rigid one, which (essentially) keeps the value of the spin fixed, even when the particle goes out of its mass shell. We let the particle go out of its mass shell, but we keep it on its "spin shell" [8]; therefore, we let the mass play a dynamical role, but we forbid this to spin. The Minkovski space plays, with respect to spin, the same role as the mass shell $\left(p^{2}=m^{2}\right)$ plays with respect to mass: it is adapted to a description of free particles, not of interacting ones.

Let us summarize this: we call the usual formulation of quantum field theory "Minkovski theory," and the present formulation "Poincare theory." The Minkovski theory contains, besides the usual axioms, a very important (though implicit) dynamical assumption.

Dynamical assumption of the Minkovski theory: the spin plays no dynamical role.

This assumption has no experimental justification. The Poincare theory is a quantum field theory without this assumption; we shall show that it treats spin on the same footing as mass, and therefore lets it play a dynamical role.

We shall give in what follows a general description of the Poincare quantum field theory. We do not try to build a definite model, but to study the general principles which (seemingly) should be satisfied by any field theory. (Otherwise stated, we have no specific dynamical principle; but this is not a special feature of the Poincare theory; furthermore, it may be that one of the obstacles which have prevented physics, up to now, to find a dynamical theory of particles, has been precisely the unjustified neglect of spin.) From this point of view, the approach used here is an axiomatic one; and we use systematically the concepts of axiomatic field theory. The emphasis, however, is not on proving theorems, but on physical interpretation. We have no new mathematical result; we merely use results obtained by the mathematicians (ref. 17, 18 and 19).

\section{Poincaré Field Theory: Relativistic Invariance}

The argument given above for replacing Minkovski space by the Poincare group was essentially classical. From a quantum point of view, it is known [9] that, because of the relation between charge (leptonic + baryonic) and spin, the Poincaré group cannot be considered alone, but we must take the non-trivial extension of this group by the gauge group corresponding to $B+L$. For simplicity's sake, however, we shall take the covering group of the (connected) Poincare group, which will be denoted by $\bar{P}$. The elements of $\bar{P}$ will be denoted by $g, h, \ldots$, or if necessary by $(a, A)$ where $a$ is a translation and $A$ a $2 \times 2$ complex unimodular matrix. The element of $\bar{P}:(0,-I)$, where $I$ is the unit matrix, is denoted by $\epsilon$ ("rotation of $2 \pi$ ").

Group $\bar{P}$ will be used to define relativistic invariance. But the fields, strictly speaking, will not be defined on group $\bar{P}$; they are defined on the manifold $\bar{P}$, without its group structure. This distinction between manifold and group is a familiar one, when one considers translations; the Minkovski space and the group of translations are two isomorphic manifolds, but a (usual) field is defined on Minkovski space, not on the translation group. This group, however, plays an important role in Minkovski field theory, because one uses the Fourier transformation, which is defined in a natural way [10] on the translation group; to do so, one chooses a reference frame in the Minkovski space, which amounts to choosing, among all the possible oneto-one mappings between the space and the group, a given one. The theory has to be invariant with respect to Poincare transformations of the Minkovski space (active transformations), and with respect to changes of reference frame, i.e., changes of the mapping between group and space (passive transformations) [11]. Here the situation is quite analogous, but as group $\bar{P}$ is not commutative, the distinction between active and passive invariance becomes more transparent (and more important).

Let us call the manifold of group $\bar{P}$, without its group structure, the $\bar{P}$ manifold. We define, then, a field as an operator-valued scalar distribution on the $\overline{\mathrm{P}}$ manifold. The elements of the $\bar{P}$ manifold will be called (incorrectly) reference frames, and will be denoted by $R_{0}, R$, etc. (This would be correct if we had the Poincare group itself instead of its covering group.)

Let $\Phi(R)$ be a field, defined on the $\overline{\mathrm{P}}$ manifold. For this field, relativistic invariance implies only active invariance; an active transformation is defined by the mapping:

$$
R \longrightarrow h R
$$

where $h$ is an element of $\bar{P}$. Relativistic invariance implies that there exists a unitary representation of $\bar{P}$, $U(h)$, such that: 


$$
U(h) \Phi(R) U(h)^{-1}=\Phi(h R) \text {. }
$$

Let us now choose an "origin" on the $\bar{P}$ manifold, i.e., a "standard reference frame" $R_{0}$. We can then write every reference frame $R$, in a unique way, as:

$$
R=g R_{0}
$$

where $g$ is an element of group $\bar{P}$. This allows us to consider the field $\Phi$ as a field on group $\overline{\mathrm{P}}$ : but this has a meaning only after we have chosen a standard frame. The field on group $\bar{P}$ will be (incorrectly) denoted by the same letter $\Phi$; thus as a consequence of (3), we define $\Phi(g)$ by:

$$
\Phi(\xi)=\Phi(R) .
$$

As a consequence of the isomorphism between group and manifold defined by (3), the active transformation (1) is mapped on a permutation of the group, called left translation:

$$
g \longrightarrow h g .
$$

The active invariance (2) writes then, for the field on the group defined by (4):

$$
U(h) \Phi(g) U(h)^{-1}=\Phi(h g) .
$$

For the field on the group, we have also passive transformations; they appear if we change the "origin" $R_{0}$ to another one $R_{0}^{\prime}$, which may be written in a unique way:

$$
R_{0}{ }^{\prime}=h R_{0} .
$$

This entails a relabeling of the manifold, i.e., we write instead of (3):

$$
R=g^{\prime} R_{0}^{\prime}=g^{\prime} h R_{0} .
$$

A given element of the manifold, $R$, previously labeled by the element $g$ of the group, is now labeled by the element $g^{\prime}=g h^{-1}$; the permutation of the group (called right translation):

$$
g \longrightarrow g^{\prime}=g h^{-1}
$$

represents a passive transformation. The passive invariance (a concept valid only for the field defined on the group) now requires that there exists a unitary representation of $\bar{P}, V(h)$, such that:

$$
V(h) \Phi(g) V(h)^{-1}=\Phi\left(g h^{-1}\right) \text {. }
$$

The Wightman functions may be defined in the usual way:

$$
F_{n}\left(g_{1}, g_{2}, \ldots g_{n}\right)=\left(\psi_{0}, \Phi\left(g_{1}\right) \Phi\left(g_{2}\right) \ldots \Phi\left(g_{n}\right) \psi_{0}\right),
$$

where $\psi_{0}$ is the vacuum state. The invariance conditions (6) and (9) write:

$$
\begin{aligned}
& F_{n}\left(g_{1}, g_{2}, \ldots g_{n}\right)=F_{n}\left(h g_{1}, h g_{2}, \ldots h g_{n}\right) \\
& F_{n}\left(g_{1}, g_{2}, \ldots g_{n}\right)=F_{n}\left(g_{1} h^{-1}, g_{2} h^{-1}, \ldots g_{n} h^{-1}\right) .
\end{aligned}
$$

The definition of statistics for the fields follows naturally from these invariance considerations; the field will be a Bose field (a Fermi field) if $U$, considered as a representation of the Poincare group itself, is onevalued (two-valued). For a Bose field, this implies $U(\epsilon)=1$, hence: 


$$
\Phi(\epsilon R)=\Phi(R) \text {. }
$$

For a Fermi field, the simplest way to have a two-valued representation is to ask that $U(\epsilon)$ (which must be a square root of the identity operator) anticommutes with the field; this implies:

$$
\Phi(\epsilon R)=-\Phi(R)
$$

On the group, these conditions write (because $\epsilon$ is in the centre of group $\bar{P}$ ):

$$
\Phi(\epsilon g)=\Phi(g)= \pm \Phi(g) .
$$

Distributions satisfying conditions of the type (13) (14) or (15) will be called even (odd) distributions.

\section{About the Spectral Condition}

In Poincare theory, the relations between the field and the Wightman functions are exactly the same as in Minkovski theory [12], and they may be expressed in the concise form given by Borchers [13]. I.e., if we are given the field, as an operator-valued distribution (the operators acting on a given Hilbert space), we may define the Wightman functions by (10); on the other hand, starting from the Wightman functions, and assuming they satisfy the positivity condition, we may define the Hilbert space and the field; the positivity condition ensures that the scalar product on the Hilbert space is positive. This is the "reconstruction theorem" $[12,13]$. The theorem also implies that, given the set of all Wightman functions $F_{n}(n=0,1$, $2 \ldots$, the representation $U$ is entirely defined. Using this fact, the Minkovski theory is able to express the spectral condition (i.e., essentially the condition that no states have negative energy or space-like energymomentum) in terms of the Wightman functions. For this, use was made initially of the Fourier transforms of the Wightman functions, which required the assumption that these functions are tempered distributions $[12,13]$. More recently, Borchers [14] has shown that this assumption is not necessary; he gives a formulation of the spectral condition which uses only analytic functions. The notion of tempered distribution, however, does not exist on group $\bar{P}$, and what can be done with analytic functions is still not clear. Therefore, we shall express the spectral condition only in an implicit way, i.e., by stating that the representation $U$ does not contain "unphysical" representations. We shall only sketch the way this condition might be expressed in terms of Wightman functions; an explicit expression involves mathematical problems which are solved only for $n=2$ (see section VII).

The spectral condition says: if we write the representation $U$ as a direct integral of multiples of irreducible representations, the only irreducible representations we find are those with positive energy, timelike or light-like energy-momentum vector, and finite spin, i.e., the representations usually denoted by $[m, s]$, where $m$ is the mass and $s$ is the spin. I.e., we have [15]:

$$
U=I \oplus \int_{0}^{\infty} d \rho(m) \sum_{s=0,1 / 2, \ldots} \nu([m, s])[m, s]
$$

Here $I$ is the identity representation, which appears once and only once (existence of a unique vacuum); $\rho$ is a (positive) measure; $\nu$ is a positive integer (possibly infinite), denoting the multiplicity of the corresponding representation.

This condition, although quite classical, is slightly more than what is called "spectral condition" in Minkovski field theory; in this theory, one requires only that the spectrum of $P_{0}$ and of $P_{\mu} P^{\mu}$ be positive. In Poincare field theory, there is no reason to give such a privileged role to the energy-momentum vector $P_{\mu}$.

Now we consider, in the state space of the field, the subspaces $\mathscr{H}_{n} ; \mathscr{H}_{n}$ is the completion of the space of states of the form:

$$
\int f\left(g_{1}, \ldots, g_{n}\right) \Phi\left(g_{n}\right) \ldots \Phi\left(g_{1}\right) d g_{1} \ldots d g_{n} \psi_{0}
$$


The subspaces $\mathscr{H}_{n}$ are invariant subspaces of the representation $U$. Let us call $U_{n}$ the restriction of $U$ to the subspace $\mathscr{H}_{n}$; the spectral condition then states that for every $n$, the direct integral decomposition of $U_{n}$ will be of the form (16). The scalar product of two vectors of $\mathscr{H}_{n}$ involves the Wightman function $W_{2 n}$; thus, the spectral condition for the subrepresentation $U_{n}$ can be in principle expressed as a property of $W_{2 n}$. Let us also consider the subspace generated by $\mathscr{H}_{n}$ and $\mathscr{H}_{n+1}$; the scalar product on this subspace involves $W_{2 n}, W_{2 n+1}$ and $W_{2 n+2}$; the spectral condition for the restriction of $U$ to this subspace can in principle be expressed as a property of $W_{2 n}, W_{2 n+1}$ and $W_{2 n+2}$.

The representation $U$ also defines the representation of the momentum and angular momentum operators $P_{\mu}, M_{\mu \nu}$ (infinitesimal generators of the $\bar{P}$ group). As shows equation (2), these generators will be represented by operators of left differentiation (also called right invariant differential operators) acting on the group manifold. Let us call these operators by the same names $P_{\mu}, M_{\mu \nu}$; we see, then, that the wave equations for a particle of mass $m$ and spin $s$ will be simply:

$$
\begin{aligned}
P_{\mu} P^{\mu} f & =m^{2} f \\
W_{\mu} W^{\mu} f & =-m^{2} s(s+1) f,
\end{aligned}
$$

where $f$ is the wave function (a scalar-valued function on $\bar{P}$ ) and:

$$
W^{\mu}=\frac{1}{2} \epsilon^{\mu \nu \rho \sigma} P_{\nu} M \rho \sigma
$$

Thus, the ambiguity of the wave equations is removed: for each type of particles there is a uniquely defined pair of wave equations (17), (18).

\section{The Two-Point Function}

We now write with more detail the properties of the two-point function, which will be important subsequently. Equation (11) implies that the two-point function $F_{2}$ depends only on $a=g_{2}^{-1} g_{1}$; for simplicity's sake, we shall write this:

$$
F_{2}\left(g_{1}, g_{2}\right)=F_{2}(\alpha)
$$

Equation (12) implies then:

$$
F_{2}\left(h a h^{-1}\right)=F_{2}(a) .
$$

This means that $F_{2}$ is a so-called central distribution: for any elements $g, h$ of $\bar{P}$, one has:

$$
F_{2}(\xi h)=F_{2}(h g) \text {. }
$$

Let us now consider the space of test functions (infinitely differentiable functions on $\bar{P}$ with compact support); let us call it $\mathscr{D}$. For any function $f$ in $\mathscr{D}$, let us define the function $\widetilde{f}$ by:

$$
\tilde{f}(g)=\overline{f\left(g^{-1}\right)} \text {. }
$$

Let us denote the image of the test function $f$ by the distribution $F$ (usually written symbolically $\left.\int F(g) f(g) d g\right)$ by $F(f)$. Then the positivity condition for $F_{2}$ can be written as:

$$
F_{2} \overline{(f * \tilde{f})} \geq 0
$$
for every $f$ in $\mathscr{D}$, where the star denotes the convolution product. Let us note that for a central distribution,
one has the following explicit form of $(20)$ :

$$
F_{2}\left(f * f^{\prime}\right)=F_{2}\left(f^{\prime} * f\right) .
$$


A distribution satisfying (21) is said to be of positive type; thus, $F_{2}$ is a central distribution of positive type. The last fact allows us to define on $\mathscr{D}$ a (positive semi-definite) inner product:

$$
\left(f, f^{\prime}\right)=F_{2} \overline{\left(f * \tilde{f}^{\prime}\right)} \text {. }
$$

The reconstruction theorem then tells us how to build the subspace $\mathscr{H}_{1}$ of the state space of the theory. The prescription is the following: let us define the subset $N$ of $\mathscr{D}$, the elements of which satisfy:

$$
(f, f)=0 \text {. }
$$

By the Schwarz inequality, $N$ is a subspace; on the quotient space $\mathscr{D} / N$, the inner product inherited from (22) is positive definite; thus we may complete this quotient to a Hilbert space, which is $\mathscr{H}_{1}$.

This construction of the subspace $\mathscr{H}_{1}$ from the two-point function is exactly the same as the construction of a representation of a group from a trace on this group [16]. Indeed, group theory calls trace a central distribution of positive type; to every trace, there corresponds a unitary representation called the left representation associated to this trace, which is defined as follows: let us start from the space $\mathscr{D}$ of functions on the group, and by taking the quotient by $N$ and completing, let us build what we have called the subspace $\mathscr{H}_{1}$; an element $h$ of $\bar{P}$ is then mapped on the operator of left translation by $h$, acting on the (classes of) functions of $\mathscr{H}_{1}$. Thus, we have obtained the following result: the subrepresentation $\mathrm{U}_{1}$ is the left representation associated to the two-point function. (This last one must be considered, according to (19), as a distribution in one variable, and therefore as a trace on group $\bar{P}$.)

\section{Free Fields}

For the field to be free, it is clearly necessary that the subrepresentation $U_{1}$ should be an irreducible representation of $\bar{P}$, say $[\mathrm{m}, \mathrm{s}]$. This gives us immediately the following result: the two-point function of a free field with mass $\mathrm{m}$ and spin $\mathrm{s}$ is the character of the irreducible representation $[\mathrm{m}, \mathrm{s}][20,21]$. (Let us recall that if the representation associated to a trace is irreducible, this trace is called a character; there is a one-to-one correspondence between characters, on the one hand, and irreducible representations, defined up to a unitary equivalence, on the other hand.)

The Wightman functions of the free field are all defined in terms of its two-point function, by the following relations. For a Bose field, we have [12]:

$$
\begin{aligned}
F_{2 j+1} & =0 \\
F_{2 j}\left(g_{1}, g_{2}, \ldots g_{2 j}\right) & =\Sigma F_{2}\left(g_{k_{1}}, g \ell_{1}\right) F_{2}\left(g_{k_{2}}, g \ell_{2}\right) \ldots F_{2}\left(g_{k_{j}}, g \ell_{j}\right)
\end{aligned}
$$

where the summation goes over all partitions of the set $(1, \ldots 2 j)$ into $j$ disjoint subsets $\left(k_{i}<\ell_{i}\right.$, $\left.k_{i}<k_{i+1}\right)$.

For a Fermi field, the result is analogous [22]:

$$
F_{2 j}\left(g_{1}, g_{2}, \ldots g_{2 j}\right)=\Sigma \eta_{k l} F_{2}\left(g_{k_{1}}, g \ell_{1}\right) F_{2}\left(g_{k_{2}}, g \ell_{2}\right) \ldots F_{2}\left(g_{k_{j}}, g \ell_{j}\right)
$$

where $\eta_{k l}= \pm 1$ according to the parity of the permutation:

$$
(1, \ldots 2 j) \longrightarrow\left(k_{1}, l_{1}, k_{2}, l_{2}, \ldots k_{j}, l_{j}\right)
$$

Thus we have given a complete characterization of the free field of mass $m$ and spin $s$. Let us also note that the free field operators satisfy the field equations (17), (18).

\section{Locality}

In Minkovski field theory, the locality condition plays an important role; its physical meaning, however, has not been entirely elucidated. We shall consider this condition, not as an evident physical requirement, but as a condition which the free field happens to fulfill, and which may be written also for a general field. Wigner [23] has shown that for any scalar field, the locality property of the two-point function: 


$$
F_{2}\left(x_{1}, x_{2}\right)=F_{2}\left(x_{2}, x_{1}\right) \quad \text { if } \quad\left(x_{1}-x_{2}\right)^{2}<0
$$

is a consequence of relativistic invariance. Indeed, if $\left(x_{1}-x_{2}\right)^{2}<0$, there exists a Poincare transformation $s$ such that $s x_{1}=x_{2}, s x_{2}=x_{1}$; relativistic invariance then implies:

$$
F_{2}\left(x_{1}, x_{2}\right)=F_{2}\left(s x_{1}, s x_{2}\right)=F_{2}\left(x_{2}, x_{1}\right) .
$$

The element $s$ is, in the Poincare group, the conjugate of a rotation of $\pi$; its square is the unit element. We shall call $s$ a symmetry.

In Poincaré field theory, an analogous property exists. A symmetry in group $\bar{P}$ is a square root, not of the unit element, but of $\epsilon$, as one sees by taking the unimodular matrix corresponding to a rotation of $\pi$ about 0 z. Therefore, on $\bar{P}$ the relation which plays the role of " $x_{1}$ and $x_{2}$ are space-like separated" is:

$$
g_{1}=s g_{2}, \quad \text { with } s^{2}=\epsilon . \quad\left(\text { Hence } g_{2}=\epsilon s g_{1} .\right)
$$

Let us consider the function $F_{2}$ for such a pair $g_{1}, g_{2}$; equations (11) and (15) imply:

$$
F_{2}\left(g_{1}, g_{2}\right)=F_{2}\left(s g_{1}, s g_{2}\right)= \pm F_{2}\left(\epsilon s g_{1}, s g_{2}\right)
$$

where the upper (lower) sign holds for a Bose (Fermi) field. Hence, when equation (25) holds, we get:

$$
F_{2}\left(g_{1}, g_{2}\right)= \pm F_{2}\left(g_{2}, g_{1}\right) .
$$

This "locality property" of the two-point function is a consequence of relativistic invariance; we observe that it gives the right connection between spin and statistics.

If now we have a free field, then as a consequence of the "locality property" (26) of the two-point function, the $n$-point functions, defined by (23) or (24) respectively, have the following property: if for a given $k$,

$$
g_{k+1}=s g_{k}, \quad \text { with } s^{2}=\epsilon
$$

then one has:

$$
F_{n}\left(g_{1}, \ldots g_{k}, g_{k+1}, \ldots g_{n}\right)= \pm F_{n}\left(g_{1}, \ldots g_{k+1}, g_{k}, \ldots g_{n}\right) .
$$

This property of free fields is a consequence of relativistic invariance; for a general field, it seems a reasonable "locality condition" to assume that (27) (28) still holds for the n-point function.

\section{Spectral Representations}

In Minkovski field theory, it is known $[24,25]$ that the two-point function of a scalar field admits a spectral representation, which can be written:

$$
F_{2}(\alpha)=\int_{0}^{\infty} d \rho\left(m^{2}\right) F_{2}\left(a ; m^{2}\right) .
$$

Here $\rho\left(m^{2}\right)$ is a positive measure, called the spectral measure; and $F_{2}\left(a ; m^{2}\right)$ is the two-point function of the scalar free field of mass $m$. It has been shown by Matthews and Salam [26] that the spectral measure has (at least in simple cases) a physical interpretation; roughly speaking, in the case where the total mass of the spectral measure is finite:

$$
\frac{1}{Z}=\int_{0}^{\infty} d \rho\left(m^{2}\right)=\rho([0, \infty])<\infty,
$$


the measure $\rho([a, b])$ of an interval $[a, b]$ is $\frac{1}{Z}$ times the probability of finding a particle of mass $m$ such that:

$$
a \leq m^{2}<b
$$

(See [12] and [27] for a mathematical discussion of the cases where $\frac{1}{Z}$ is infinite.)

In particular, the $\delta$ function components in $\rho$ represent stable particles, and the peaks represent unstable particles; the larger the peak is, the larger is the mass spectrum of the corresponding particle, the shorter its lifetime.

In Poincare theory, we find naturally spectral representations which generalize the Kallèn-Lehmann representation (29). We have seen in Section II that a two-point function is a trace, or central distribution of positive type. It is known that, for groups of a large class, including the $\bar{P}$ group, every trace has a unique spectral representation [28] which for the present case writes:

$$
F_{2}(a)=\sum_{s} \int_{0}^{\infty} d \rho\left(m^{2}, s\right) F_{2}\left(\alpha ; m^{2}, s\right)+F_{2}^{(\mathrm{un})}(a)
$$

Here $\rho\left(m^{2}, s\right)$ is a generalized spectral measure, which depends not only on mass, but also on spin; $F_{2}\left(a ; m^{2}, s\right)$ is the two-point function of the free field of mass $m$ and $s p i n s$, i.e., the character of the corresponding representation. For a Bose field (a Fermi field), we know by equation (15) that the field is an even function (an odd function); hence $F_{2}(a)$ has the same property. But $F_{2}\left(\alpha ; \mathrm{m}^{2}, \mathrm{~s}\right)$ also is an even or odd function, if $s$ is integer of half-integer. Therefore, in (30) the summation goes over all integral (halfintegral) values of $s$, if the field is a Bose (a Fermi) field.

$F_{2}{ }^{(\text {un) }}(a)$ represents the unphysical part of the trace $F_{2}, i . \Theta$. , the part whose spectral measure is concentrated on the unphysical irreducible representations of $\bar{P}$ (space-like momentum, negative energy, infinite spin, zero momentum). The argument to put this part equal to zero is the following: to every trace corresponds a representation, and the spectral measure of the trace is a spectral measure for the direct integral decomposition of the representation. In our case, the representation corresponding to $F_{2}$ is the subrepresentation $U_{1}$ of $U$, i.e., the restriction of $U$ to the subspace $\mathscr{H}_{1}$. The spectral condition implies that $U_{1}$ has no component on the unphysical irreducible representations; hence, the same is true for the trace $F_{2}$, and the spectral condition implies, for the two-point function, the condition:

$$
F_{2}^{\text {(un) }}=0 \text {. }
$$

The spectral representation (30) has an important physical significance. It shows, first, that a given field may be associated in a simple way to particles of different spins; if the spectral measure $\rho\left(\mathrm{s}, \mathrm{m}^{2}\right)$ contains a $\delta$ function $\delta_{s s_{0}} \delta\left(m^{2}-m_{0}^{2}\right)$, there is a stable particle of mass $m_{0}$, spin $s_{0}$; if $\rho$ has a peak around $m_{0}, s_{0}$, there is an unstable particle of (average) mass $m_{0}$, (average) spin $s_{0}$; this particle will have a mass spectrum of finite width, and also a spin spectrum of finite width, which, according to the general principles of quantum mechanics, must indeed be the case, as Beltrametti [31] has remarked. All these particles, stable or unstable, are dynamically related, as they are represented by the same field. This dynamical relation may also be expressed in another way: for each value of $\mathrm{m}^{2}$, the measure $\rho\left(s, m^{2}\right)$ defines a spin spectrum. This spin spectrum function of the mass is a generalization of the notion of Regge trajectory. Indeed, a Regge trajectory [32] is a complex spin function of the mass; but a complex spin means really a spin spectrum of a particular form (a "Breit-Wigner form"), as shows the expression of Legendre functions of complex index in terms of the functions of integral index [33]:

$$
P_{\lambda}^{m}(\cos \theta)=\frac{\sin \lambda \pi}{\pi} \sum_{s=0}(-1)^{s}\left(\frac{1}{\lambda-s}-\frac{1}{\lambda+s+1}\right) P_{s}^{m}(\cos \theta) .
$$

The amplitude of the spin spectrum is the expression between brackets; if $\operatorname{Re} \lambda \gg 1$, the only important term is the first one, $(\lambda-s)^{-1}$, a function of $s$ of the Breit-Wigner form, with average value $\operatorname{Re} \lambda$ and width $\operatorname{Im} \lambda$. 
Of course, this form is a very special one for a spin spectrum; it is likely that, in real cases, it is valid only when one is not too far from the center of the peak. From the point of view of the Poincare theory, the notion of Regge trajectory appears as a special form of the spectral measure $\rho\left(s, m^{2}\right)$.

A related point for the interpretation of the spectral measure is the following: let us consider a Minkovski field, whose spectral measure contains a $\delta\left(m^{2}-m_{0}{ }^{2}\right)$ and a continuous part. This last part is usually interpreted with the notion of virtual particle, or of particle which goes out of its mass shell. As the Minkovski spectral measure does not depend on spin, the particle will remain on its spin shell, even when it goes out of its mass shell. (See footnote [8]; what we say now is strictly true for the cases, most important in practice, of scalar and spinor fields.) A Poincare spectral measure, containing a $\delta_{s s_{0}} \delta\left(m^{2}-m_{0}^{2}\right)$ and a continuous part, may be interpreted in an analogous way: if $\rho\left(s, m^{2}\right)$ is not identically zero when $s \neq s_{0}$, the particle will go out of its spin shell when it goes out of its mass shell. This is a necessary condition for the spin to be allowed to play a dynamical role.

\section{Conclusion}

In what respect is the Poincare theory inequivalent to the Minkovski theory? This question may be viewed from two sides. On the one hand, we may adopt a strict axiomatic point of view. We then define a field theory as a theory of an enumerable set of fields [34]; all the physical results of the theory are considered to be given by the $S$-matrix, and the equivalence problem reads: given a Poincare field (or a small number of Poincare fields), does there exist a (possibly infinite) set of Minkovski fields, satisfying the usual axioms, and having the same $S$-matrix? The answer is of course not known, as the existence problem of field theory is not solved; but it may very well be "yes."

On the other hand, we may consider a field (or a set of fields), not only as an interpolating field for a given $S$-matrix, but as a concept which expresses physical reality also in a more direct way. This is the point of view of Matthews and Salam [26], when they define unstable particles as peaks in the spectral function of the two-point function; this is also the point of view of Schwinger [36] about unstable particles; (note that Schwinger uses Green's functions to formulate this idea). This point of view is less remote from the particles one sees in laboratories, almost all of which are unstable particles. (Even for the so-called stable particles, we know experimentally only a lower bound for their life-time [35].)

If we adopt this "physical" point of view about quantum field theory, we have, besides the usual axioms, some additional requirements to satisfy. We do not want an infinite set of fields (if this may be avoided), but, as far as possible, a small set of fields. Each field must describe dynamically related particles - stable or unstable. But we know (as Regge pole theory has pointed out) that dynamically related particles frequently have different spins. Therefore, a given field must be able to have, in the spectral measure of its two-point function, peaks for different values of the spin - and this requires Poincare theory.

From this "practical" point of view, one may conjecture that the answer to the equivalence problem will probably be the following: both theories are equivalent (exactly or approximately) when the spin plays only a negligible dynamical role, i.e., when the slope of the Regge trajectories is small. For instance, this is probably the case in electrodynamics. But when the dynamical role of the spin is important, i.e., when the slope of the trajectories is appreciable, the two theories are no more equivalent, because the Minkovski theory contains the implicit dynamical assumption mentioned in Section I.

After these hypothetical remarks, we would like finally to draw attention to another aspect of Poincaré theory, namely the fact that it uses the spectral representation (30) for the trace $F_{2}$ on the $\bar{P}$ group. Let us compare (30) with the direct integral decomposition of the subrepresentation $U_{1} ; U_{1}$ is multiplicity free, i.e., (ref. 19) each irreducible component has a multiplicity one; therefore:

$$
U_{1}=\sum_{s} \int_{0}^{\infty} d \rho\left(m^{2}, s\right)\left[m^{2}, s\right]
$$

The difference between (30) and (33) is that the measure $\rho$ in (30) is uniquely defined, but the measure $\rho$ in (33) is defined up to an equivalence. (Two measures are said to be equivalent when they have the same null sets.) Accordingly, the trace $F_{2}$ and the characters $F_{2}(m, s)$ in $(30)$ are uniquely defined distributions, but the representations $U_{1}$ and $\left[\mathrm{m}^{2}, \mathrm{~s}\right]$ in (33) are defined up to an equivalence. Therefore, (30) contains much 
more physical information than (33); to each trace there corresponds a representation, but a representation has many traces [19].

Thus, in the simple case of multiplicity free representations, the traces turn out to be the right tool for realizing the program recently defined by Dirac [37]: "to set up a suitable representation of the inhomogeneous Lorentz group corresponding to physical reality."

\section{Acknowledgements}

The author is grateful, for their patient mathematical help, to F. Bruhat, J. Dixmier, J. P. Serre and M. Zerner. He has benefitted from fruitful discussions with C. Bouchiat, K. Chadan, M. Fierz, G. Flamand, R. Hakim, D. Kastler, J. Lascoux, J. M. Lévy-Leblond, L. Michel, E. de Rafaël. He also thanks H. J. Borchers, R. Jost, H. Lehmann, A. S. Wightman, for pointing out references or results of the axiomatic field theory.

\section{References}

1. An explicit statement of this idea may be found, for instance, in the paper of K. HEPP: Helv. Phys. Acta 37, 55 (1964).

2. To the author's knowledge, a group manifold has been used explicitly as a configuration space for the first time in Wigner's paper on classical multiple scattering; E. P. WIGNER: Phys. Rev. 94, 17 (1954). Wigner mentions the possibility of using the extra variables, which appear in the group formulation, to describe the spin of the particles.

3. It has been remarked by Segal that the Minkovski space "may well have hardly more operational significance for microscopic elementary particle theory than the ether." I. E. SEGAL: Phys. Rev. 109, 2191 (1958). See p. 2197.

4. E. P. WIGNER: Zeits. f. Phys. 124, 665 (1948). See in particular footnote 1, p. 668.

5. M. FIERZ: Helv. Phys. Acta 12, 3 (1938). For a general discussion of wave equations, see ref. 4.

6. S. S. SCHWEBER: An introduction to relativistic quantum field theory. (Evanston, Ill. 1961).

7. This situation has been recently criticized, from a different point of view, by S. WEINBERG: Phys. Rev Rev. 133, B 1318 (1964).

8. When there are redundant components, the formalism allows in fact some wandering out of the spin shell, but only towards the lower spin values, and in a very special way.

9. F. LURÇAT, L. MICHEL: Nuov. Cim. 21, 574 (1961).

10. See, for instance, A. WEIL: L'intégration dans les groupes topologiques et ses applications (Paris, 1953).

11. The distinction between active and passive invariance is explained, from a slightly different point of view, by A. S. WIGHTMAN: Nuov. Cim. Suppl. 14, 81 (1959).

12. A. S. WIGHTMAN: Phys. Rev. 101, 860 (1956).

13. H. J. BORCHERS: Nuov. Cim. 24, 214 (1962).

14. H. J. BORCHERS: Nuov. Cim. (to be published).

15. A. S. WIGHTMAN: L'invariance dans la mécanique quantique relativiste. In: Relations de dispersion et particules élémentaires (C. de Witt, R. Omnès, ed.) (Paris, 1960).

16. We use the terminology of Godement and Guichardet (see references 17 and 18). Mackey (ref. 19) calls character what we call a trace, and irreducible character what we call a character.

17. R. GODEMENT: Ann. Math. 59, 47 and 63 (1954).

18. A. GUICHARDET: Ann. Inst. Fourier 13, 1 (1963).

19. G. W. MACKEY: Bull. Amer. Math. Soc. 69, 628 (1963).

20. In his paper about models of field theory with finite invariance groups (Journ. Math. Phys. 5, 155 (1964)), H. JOOS has found that the characters of the finite group, or rather the restriction of these characters to the group which plays there the role of the translation subgroup, correspond to the functions $\Delta^{+}$and $\Delta^{-}$of field theory.

21. H. LEHMANN has kindly informed me that $\mathrm{H}$. Joos and a collaborator are now computing explicitly the characters of the group $\bar{P}$.

22. W. SCHMIDT, K. BAUMANN: Nuov. Cim. 4, 860 (1956). 
23. E. P. WIGNER, in: La théorie quantique des champs. (Solvay meeting 1961), New York, London and Brussels, 1962. p. 168

24. G. KALlÈn: Helv. Phys. Acta 25, 416 (1952).

25. H. LEHMANN: Nuov. Cim. 11, 342 (1956).

26. P. T. MATTHEWS, A. SALAM: Phys. Rev. 112, 283 (1958); 115, 1079 (1959).

27. O. STEINMANN: Journ. Math. Phys. 4, 583 (1963).

28. See ref. (19) for a simple statement of the relevant results, and references (17), (18) for proofs. The class of groups in question is the class of type I groups; it has been shown by Wigner (ref. (29)) and Mackey (ref. (30)) that the $\bar{P}$ group is a type I group.

29. E. P. WIGNER: Ann. Math. 40, 149 (1939).

30. G. W. MACKEY: Group representations in Hilbert space. Appendix to: I. E. Segal, Mathematical problems of relativistic physics. Amer. Math. Soc. 1963.

31. E. G. BELTRAMETTI: Nuov. Cim. 25, 1393 (1962).

32. G. F. CHEW, S. C. FRAUTSCHI: Phys. Rev. Lett. 7, 394 (1961); 8, 41 (1962).

33. W. MAGNUS, F. OBERHETTINGER: Formulae and theorems for the functions of mathematical physics. New York, 1954. p. 73.

34. D. RUELlE: Helv. Phys. Acta 35, 147 (1962).

35. For the electron, see: G. FEINBERG, M. GOLDHABER, Proc. Nat. Acad. Sci. 45, 1301 (1959); for the proton, see: G. BACKENSTOSS, H. FRAUENFELDER, B. HYAMS, L. KOESTLER, P. MARIN: Nuov. Cim. 16, 749 (1960).

36. J. SCHWINGER: Ann. Phys. 9, 169 (1960).

37. P. A. M. DIRAC, in: La théorie quantique des champs (see reference 23), pp. 93 and 167. See also P. A. M. DIRAC: Rev. Mod. Phys. 34, 592 (1962). 